\title{
COLOR FEATURE WITH SPATIAL INFORMATION EXTRACTION METHODS FOR CBIR: A REVIEW
}

\author{
Sarmad T. Abdul-samad 1 \\ ${ }^{1}$ AL-Nahiriain University// Computers Science Department \\ Baghdad, Iraq \\ sarmad.thaer991@rdd.deu.iq
}

\author{
Sawsan Kamal ${ }^{2}$ \\ ${ }^{2}$ AL-Nahiriain University// Computers Science Department \\ Baghdad, Iraq \\ skt@sci.nahra1inuniiv.edu.iq
}

\begin{abstract}
Inn then last two decades the Content Based Image Retrieval (CBIR) considered as one of the topic of interest for the researchers. It depending one analysis of the image's visual content which can be done by extracting the color, texture and shape features. Therefore, feature extraction is one of the important steps in CBIR system for representing the image completely. Color feature is the most widely used and more reliable feature among the image visual features. This paper reviews different methods, namely Local Color Histogram, Color Correlogram, Row sum and Column sum and Colors Coherences Vectors were used to extract colors features taking in consideration the spatial information of the image.
\end{abstract}

Keywords: Spatial Features, Color Histogram, Color Correlogram, Color Coherence Vector

\section{INTRODUCTION}

In the last years, the development of the multimedia applications led to widespread of digital images. Also, the developing of the images' sharing unlimited number of images via social media every day. However, managing and organizing these digital images present a problem. Thus, the concepts of indexing and retrieval were introduced to overcome this issue. Indexing relates to "how images are store in database to retrieve them (through querying) more efficient", whereas Retrieval relates to "how to retrieve images that are most relevant to the query image from images in database" $[1,2,3]$.

At the First, Text -Based Image Retrieval (TBI R) are used to achieve the image retrieval task. It's depend one metadata that related to each image and the retrieving of image task done by using traditional query techniques "using keyword". This method works well with small digital images databases but, it has low efficiency with huge database. The most important problem in TBIR is different users use different words to describe the same image (subjectivity of the human). This problem negatively affects the efficiency of the text-based image search, so, a need for more efficient image retrieval system is appeared. The needed system must perform an automatic indexing and retrieving. Therefore, the second method depending on image content for indexing and retrieving. Therefore, this method is generally known as Content -Based Image Retrieval (CB IR) [4] .

\section{CONTENTS-BASED IMAGES RETRIEVALS}

CB IR was introduced in the 1990's. It depending on analysis of the image's visual content which can be analyzed by extracting image features such as color, texture and shape that are called low level features [5]. In order to design and implement generic CBIR applications, both advanced algorithms in image understanding field and advances in computer hardware is needed, which are unrealized at this time [6,7]. Therefore, most efforts are directed to a specific CBIR applications [6,7]. A wide range of CBIR applications varied from personal to medical diagnoses, crime prevention, education, military and many other applications [8]. Figure 1 shows CBIR system steps.

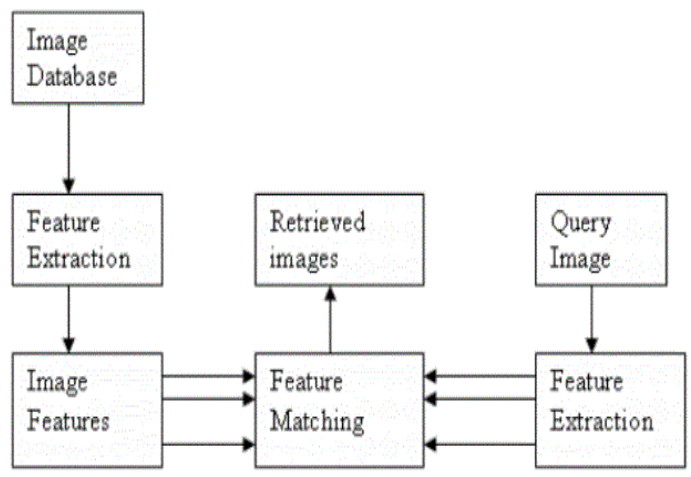

Figure (1): CBIR System Diagram [9] 
The main steps in CB IR are:

Features Extraction - In this step the features of images are extracted. The definition of features is mention before which are color, texture and shape. Features are stored in form multi-dimensional vectors of real values.

Indexing - The task of organizing the images' database using a specific indexing structure for retrieval.

Retrieval - The retrieval takes can be performed more effective on the structured database.

\section{COLOR FEATURE}

Color feature considered as one of the most widely used visual features in CBIR, and can visually be recognized by the humans. Color results from the light interaction with a human eyes and brain. Human mostly based on color feature to distinguish the images. It is also used as a powerful descriptor that simplifies object identification [10]. Color feature are easy to extract and it is found to be effective for indexing and searching colored images. To extract color feature from the image, a proper color space (also called color module) such as RGB (Red, Green, and Blue), HSV (Hue, Saturation, and Value) must be selected and also an effective descriptor should be determined [11].

\section{COLORS FEATURES EXTRACTIONS METHODS}

\section{Local Colors Histogram (LCH)}

The most widely used technique for color feature extracting of an image is colors histogram. It represents the image from a different perspective. It represents the frequency distribution of color's bins in an image. It count s simila $r$ pixe ls and store it. There are tw o types of color histogram. Globa 1 colo $\mathrm{r}$ histograms and loca 1 col or histograms. Co lor histogr am is proposed as a global color descriptor which analyze every statistica 1 color frequency in an image. Local color histogram focuses the colo $r$ distribution of regions. The spatial distribution of pixel is taken in calculation when using $\mathrm{LCH}$, which is not calculated when using Global colors histogram. It calculated by segments the image into many segments or fix parts and then, the histogram is calculated these segments. Image will then be represented by whole histograms [4].

\section{Color Correlorgram}

Spatial information of the extracted feature is the main drawback of the color histogram. For example, all the images shown in figure 2 have the same color proportion but, different spatial distribution Correlation histogram (correlogram) tries to fix this histogram's drawback by taking the spatial correlation of color distribution into account, and shows how the spatial correlation between pairs off colors are changing with distance [13].

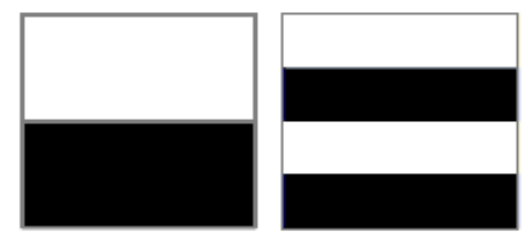

Figure (2): Images having same color proportions but, different spatial distribution [12].

A color correlogram can be represented as a table indexed by color pairs $(i, j)$ where, the $d^{\text {th }}$ entry specified the probably of finding a pixel with I color at a distains $d$ form the pixel with $\mathrm{j}$ color in the image.

Let [D ] denote the set of distances $\left\{\mathrm{d}_{1}, \ldots, \mathrm{d}_{\mathrm{D}}\right\}$. Then the color correlogram for the image $\mathbf{I}$ for color pair $\left(\mathrm{c}_{\mathrm{i}}, \mathrm{c}_{\mathrm{j}}\right)$ at a distance $\mathbf{d}$ can be denoted as [14]:

$C_{c_{i, c_{j}}}^{d}(I)=\operatorname{prob}_{P 1 \in I_{c(i)}, P 2 \in I}\left[P_{2} \in I_{c(j)}|| P_{1}-P_{2} \mid=d\right]$

Where:

$P_{1}, P_{2}$ are the probabilities of the color occurrence

\section{Color Coherence Vector}

In the color coherence vector (CCV) the images' pixels are partitioned according to their spatial coherence into two groups, i.e., coherent or in-coherent . If those pixels belong to a large uniformed color region, it's called coherence, otherwise it in-coherence. After calculating the CCV separate histograms can be produced for both coherent and incoherent pixels. CCV having some problem, but the main problem and the most time consuming is the computation of three dimensional index vector. To calculate the index vector, all image's pixels must be compared with all of its adjacent pixels to find out whether this pixel is belong to coherence or incoherence group. Using CCV 
success with CBIR systems due to its additional spatial information [15] [16].

\section{a. Row Sum and Column Sum}

Row sum and column sum is another type of color spatial features. For any two similar images, the row sum and column sum are approximately equal. The row sum for any images can be calculated as follow:

$$
\operatorname{Row} \operatorname{Sum}(i)=\sum_{j=1}^{N} I(i, j)
$$

Where, $\mathrm{M}=$ Rows' number in an image

$\mathrm{N}=$ Columns' number in an image

$\mathrm{i}=1,2,3 \ldots \ldots . \mathrm{M}$

Column sum can also represent the distribution of pixel value in the image, therefore computing column sum, can determine the flow of pixel value distribution in an image. It can be calculated as follows:

$$
\operatorname{ColumnSum}(i)=\sum_{i=1}^{M} I(i, j)
$$

Where, $\mathrm{M}=$ Rows number in an image

$\mathrm{N}=$ Columns' number in an image

$\mathrm{j}=1,2,3 \ldots \ldots . \mathrm{N}$

Row sum and column sum can be calculated for each channel of the RGB color space. For example, if the image size is $(256 * 256)$ a 256 value for each image row and 256 value for each image column are calculated [17].

b. Color structure descriptors (CSD)

The CS D represents color content information (color histogram in addition to information about them structure of its content i.e. localized color distribution using structuring window ). The performance of CS D relies on the size and structure of the window, which are difficult to specify The C SD is connected to the double-coned HM MD color space which is quantized non-uniformly into $32,64,128$ or 256 bins, this feature guarantees cementing the color structure information into the CS D, this is achieved $\mathrm{b}$ y considering all colors inn a structuring window which slides over the image, instead of considering individual pixel separately. For example, bin i of the histogram shows how many times the structuring element contains at least one pixel with color i. If the window is of size 1 pixel [18] [19].

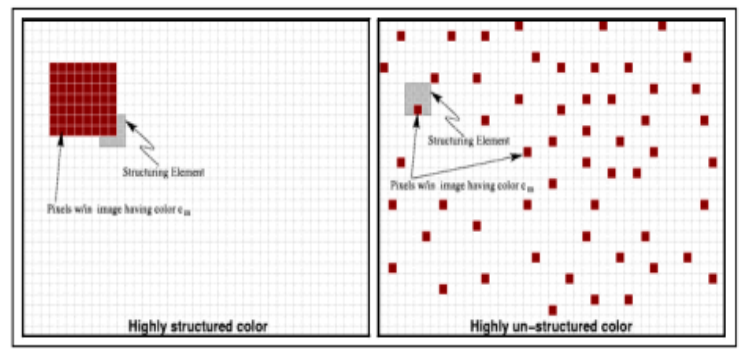

Figure (3)- two images have same histogram but the right one has much more gray component in CSD description [20].

\section{COMPA RATIVE ANA LYSIS}

A comparative analysis of the color feature extraction techniques with their advantages and disadvantages are shown in table 1 .

TA BLE 1 - A COMPA RATIVE ANA LYSIS OF COL OR

\begin{tabular}{|c|c|c|c|}
\hline Feature & Advantage & Disadvantage & $\begin{array}{c}\text { Published } \\
\text { latest } \\
\text { papers }\end{array}$ \\
\hline $\begin{array}{c}\text { Local Color } \\
\text { Histogram }\end{array}$ & $\begin{array}{l}\text { It is simple to } \\
\text { use and faster } \\
\text { than the other } \\
\text { method in } \\
\text { computations }\end{array}$ & $\begin{array}{c}\text { It gives } \\
\text { different result } \\
\text { for retrieval } \\
\text { task when the } \\
\text { image's } \\
\text { orientation, } \\
\text { and position or } \\
\text { scales are } \\
\text { changed. }\end{array}$ & [4] [21] \\
\hline $\begin{array}{c}\text { Color } \\
\text { Correlogram }\end{array}$ & $\begin{array}{l}\text { It is simple to } \\
\text { compute and } \\
\text { may be used } \\
\text { to distinguish } \\
\text { images }\end{array}$ & $\begin{array}{l}\text { It is slow } \\
\text { computations } \\
\text { and high } \\
\text { dimensionality }\end{array}$ & $\begin{array}{c}\text { [22] [23] } \\
{[24]}\end{array}$ \\
\hline $\begin{array}{c}\text { Color } \\
\text { Coherence } \\
\text { Vector }\end{array}$ & $\begin{array}{l}\text { It gives good } \\
\text { spatial } \\
\text { information } \\
\text { about color } \\
\text { distribution } \\
\text { into the } \\
\text { image }\end{array}$ & $\begin{array}{l}\text { It } \\
\text { computations } \\
\text { is highly cost }\end{array}$ & [25] \\
\hline
\end{tabular}
FEAT URE EXTRAC TION TECHN IQUES 


\begin{tabular}{|c|c|c|c|}
\hline $\begin{array}{c}\text { Row sum } \\
\text { and Column } \\
\text { sum }\end{array}$ & $\begin{array}{c}\text { It is easy and } \\
\text { faster } \\
\text { computations }\end{array}$ & $\begin{array}{c}\text { It has different } \\
\text { size feature } \\
\text { vector's size } \\
\text { for different } \\
\text { sized images }\end{array}$ & {$[16]$} \\
\hline $\begin{array}{c}\text { Color } \\
\text { Structure } \\
\text { descriptor }\end{array}$ & $\begin{array}{c}\text { Gives spatial } \\
\text { info }\end{array}$ & $\begin{array}{c}\text { It is effected } \\
\text { by rotation } \\
\text {,noise and } \\
\text { scale }\end{array}$ & {$[26]$} \\
\hline
\end{tabular}

An important issue while selecting the color feature extraction methods are storage space required, scalability, rotation invariant, computational time required and its feasibility, and efficient in storage space and time . complexity parameters". Histogram is easy to compute , but isn't robust and unique whereas using local color histogram solve a part of the problem since it gives a spatial information about each region of the image, and this approach is also computationally an easy approach. The correlogram solving the problem of histogram but it will increase the size of features' vector and effect the storage, and this will take more time. So, using the auto-correlogram will give approximately similar results and similar effects on the performance of the system with less values. In row sum and column sum the images must have same size in order to produce a same number of rows and columns for each image, these prove that these features are effected by the image size. Color coherence vector solve all the problems the previously discussed but it's computationally cost, to calculate the index vector, all image's pixels must be compared with all of its adjacent pixels to find out whether this pixel is belonging to coherence or incoherence group which is time consuming but success with CBIR systems due to its additional spatial information as mentioned in previous sections.

\section{Conclusion}

CBIR is one of the most important research topic in pattern recognition, image processing and computer vision fields of study. The CBI R goal is to retrieve relevant images from images collected in database that can be done by measures the similarities between the query image and the database's image s. "It is generally base on analyzing the visual content of the image depending on three basic low level features, which are: color, texture and shape, and the color is the most important one among them". Some of methods used to extract color feature are discussed in this paper. The selection of a method depends on its functionality for a specific purpose.

\section{REFERENCES}

[1] Narasimha, Yadav RP, L. K. Pavithra, and Sharmila T. Sree "Analysis of Supervised and Unsupervised Learning in Content Based Multimedia Retrieval." In 2018 International Conference on Computer, Communication, and Signal Processing (ICCCSP), pp. 1-5. IEEE, 2018.

[2] Putzu, Lorenzo, Luca Piras, and Giorgio Giacinto. "Ten Years of Relevance Score for Content Based Image Retrieval." In International Conference on Machine Learning and Data Mining in Pattern Recognition, pp. 117-131. Springer, Cham, 2018.

[3] Najeeb, Rana F., and Ban N. Dhannoon. "Classification for intrusion detection with different feature selection methods: a survey (20142016)." International Journal of Advanced Research in Computer Science and Software Engineering 7, no. 5 (2017).

[4] Lonarkar, Vishal, and B. Ashwath Rao. "Content-based image retrieval by segmentation and clustering." In 2017 International Conference on Inventive Computing and Informatics (ICICI), pp. 771-776. IEEE, 2017.

[5] Qi, Yonggang, Yi-Zhe Song, Honggang Zhang, and Jun Liu. "Sketch-based image retrieval via siamese convolutional neural network." In 2016 IEEE International Conference on Image Processing (ICIP), pp. 2460-2464. IEEE, 2016.

[6] Pavlidis, T. "Limitations of cbir." In Plenary talk, the Nineteenth International Conference on Pattern Recognition. 2008.

[7] Penatti, Otávio AB, Eduardo Valle, and Ricardo da S. Torres. "Comparative study of global color and texture descriptors for web image retrieval." Journal of visual communication and image representation 23, no. 2 (2012): 359-380.

[8] Singh, Bohar, and Mrs Mehak Aggarwal. "A Review On Content Based Image Retrieval." INTERNATIONAL JOURNAL OF COMPUTERS \& TECHNOLOGY 17, no. 2 (2018): 7226-7235.

[9] Dureja, A., \& Pahwa, P. Image retrieval techniques: a survey. International Journal of Engineering \& Technology, 7(1.2), 215219. (2018)

[10] Sundara Vadivel, P., et al. "An efficient CBIR system based on color histogram, edge, and texture features." Concurrency and Computation: Practice and Experience 31.12 (2019): e4994.

[11] Doimari, Dweep Chand, Saif Khusro, and Sanu Anand. "Content Based Image Retrieval using RGB to HSV conversion." (2018).

[12] HAZRA, Dipankar. Retrieval of color image using color correlogram and wavelet filters. In: International Conference on advances in computer engineering. 2011.

[13] Soni, Devyani, and K. J. Mathai. "An efficient content based image retrieval system based on color space approach using color histogram and color correlogram." In 2015 Fifth International Conference on Communication Systems and Network Technologies, pp. 488-492. IEEE, 2015.

[14] Mistry, Yogita, D. T. Ingole, and M. D. Ingole. "Content based image retrieval using hybrid features and various distance metric." Journal of Electrical Systems and Information Technology (2017).

[15] Shukla, Jahnavi, and Jignesh Vania. "A survey on CBIR features extraction techniques." International Journal of Engineering and Computer Science 3, no. 12 (2014): 9555-9559.

[16] Ravani, Reza, Mohamad Reza Mirali, and Maryam Baniasadi. "Parallel CBIR system based on color coherence vector." In 17th 
[17] ElAdel, Asma, Ridha Ejbali, Mourad Zaied, and Chokri Ben Amar. "A hybrid approach for Content-Based Image Retrieval based on Fast Beta Wavelet network and fuzzy decision support system." Machine Vision and Applications 27, no. 6 (2016): 781-799.

[18] Mane, Pranoti, and Dr NG Bawane. "Comparative performance evaluation of edge histogram descriptors and color structure descriptors in content based image retrieval." IJCA Proceedings on NCIPET 6 (2013): 59.

[19] Zhang, Dengsheng, Md Monirul Islam, and Guojun Lu. "A review on automatic image annotation techniques." Pattern Recognition 45, no. 1 (2012): 346-362.

[20] Abdelali, Abdessalem Ben, et al. "A study of the color-structure descriptor for shot boundary detection." International Journal of Sciencies and Techiques of Automatic control and computer engineering (2009): 956-971

[21] Nazir, Atif, et al. "Content based image retrieval system by using HSV color histogram, discrete wavelet transforms and edge histogram descriptor." 2018 International Conference on Computing, Mathematics and Engineering Technologies (iCoMET). IEEE, 2018.
[22] Bu, Hee-Hyung, et al. "Content-based image retrieval using combined texture and color features based on multi-resolution multidirection filtering and color autocorrelogram." Journal of Ambient Intelligence and Humanized Computing (2019): 1-9.

[23] Vinayak, Vandana, and Sonika Jindal. "CBIR system using color moment and color auto-Correlogram with block truncation coding." International Journal of Computer Applications 161.9 (2017):1-7

[24] Mustaffa, Mas Rina, Fatimah Ahmad, and Shyamala C. Doraisamy. "Multi-resolution joint auto correlograms for content-based image retrieval." Advanced Science Letters 23.6 (2017): 5370-5374.

[25] Singh, Jyotsna, et al. "Content Based Image Retrieval using Gabor Filters and Color Coherence Vector." 2018 IEEE 8th International Advance Computing Conference (IACC). IEEE, 2018.

[26] Mane, Pranoti, and Dr NG Bawane. "Comparative performance evaluation of edge histogram descriptors and color structure descriptors in content based image retrieval." IJCA Proceedings on NCIPET 6 (2013): 5 9. 